\title{
Immune Complex Type Crescentic Glomerulonephritis Accompanied with Perinuclear Anti-Neutrophil Cytoplasmic Antibodies
}

\author{
Atsushi Komatsuda, Tadashi Yasuda, Hideki Waku, Hirokazu Imai, Akira B Miura, \\ Masafumi SAKUYAMA*, Takashi FuKUDA* and Yasushi NAKAMOTO**
}

\begin{abstract}
A 70-year-old male developed rapidly progressive glomerulonephritis syndrome with serum perinuclear anti-neutrophil cytoplasmic antibodies (P-ANCA). A renal biopsy showed diffuse crescentic glomerulonephritis. Immunofluorescence microscopy revealed 2+ granular staining of IgG over the mesangial area and along glomerular capillary walls. Electron microscopy showed scattered deposits in the paramesangial area. These morphologic findings were consistent with those of immune complex type crescentic glomerulonephritis (IC-CGN). Serum C3, C4, and CH50 were within normal limits, and circulating immune complexes were not detected by C1q-binding assay, but both P-ANCA and anti-myeloperoxidase antibodies were positive. A possible relation of P-ANCA to IC-CGN is discussed.
\end{abstract}

(Internal Medicine 32: 387-390, 1993)

Key words: ANCA-associated renal diseases

\section{Introduction}

Rapidly progressive glomerulonephritis is associated with primary glomerular diseases, post-infectious diseases, and multisystemic diseases. The pathogenetic mechanisms causing crescent formation have been clarified by immunoserological methods $(1,2)$. Recently, anti-neutrophil cytoplasmic antibodies (ANCA) have been recognized as a serological marker for systemic necrotizing arteritis and idiopathic necrotizing and crescentic glomerulonephritis. The positive reaction of ANCA can be found in a variety of diseases, such as Wegener's granulomatosis, polyarteritis nodosa, Churg-Strauss syndrome, and other collagen diseases including systemic lupus erythematosus (SLE). These are called ANCA-associated diseases (3-5). In idiopathic crescentic glomerulonephritis, 70 to $80 \%$ of patients with pauci-immune type crescentic glomerulonephritis show ANCA, especially perinuclear-ANCA (P-ANCA) (6). ANCA are also found to coexist in some patients with anti-glomerular basement membrane antibodymediated type crescentic glomerulonephritis $(30$ to $40 \%)(7,8)$. However, it is reported that ANCA are consistently negative in patients with immune complex type crescentic glomerulonephritis (1).
In this report, we describe a 70-year-old man who had rapidly progressive glomerulonephritis due to crescentic glomerulonephritis with immune complex deposits, and had serum P-ANCA. Possible pathogenetic mechanisms of crescentic formation in this case are also discussed.

\section{Case Report}

A 70-year-old Japanese man was admitted to Akita Rohsai Hospital because of facial edema and non-bloody productive cough on June 28,1991 . He had been well until one month prior to admission, when he noticed facial edema and had a cough with sputum.

On admission, his body temperature was $36.0^{\circ} \mathrm{C}$, pulse rate $66 / \mathrm{min}$, respiratory rate $16 / \mathrm{min}$, and blood pressure $194 / 114$ mmHg. A physical examination revealed moderate pitting edema in both legs and mild edema in the face. Coarse crackles were heard throughout both lower lung fields. The heart sounds were normal, but a mild hepatomegaly was found. The neurologic examination was negative.

The erythrocyte sedimentation rate was $102 \mathrm{~mm} / \mathrm{h}, \mathrm{C}-\mathrm{reac}-$ tive protein $4.7 \mathrm{mg} / \mathrm{dl}$, and anti-streptolysin $\mathrm{O}$ titer $30.6 \mathrm{Todd}$. The erythrocyte count was $398 \times 10^{4} / \mu 1$, hemoglobin $11.0 \mathrm{~g} / \mathrm{dl}$,

From the Third Department of Internal Medicine, Akita University School of Medicine, Akita, *the Department of Internal Medicine and Urology, Akita Rohsai Hospital, Ohdate, and **the Department of Internal Medicine, Mitaka Kitaguchi Hospital, Musashiro

Received for publication October 9, 1992; Accepted for publication March 16, 1993

Reprint requests should be addressed to Dr. Atsushi Komatsuda, the Third Department of Internal Medicine, Akita University School of Medicine, 1-1-1 Hondo, Akita 010, Japan 
hematocrit $33.5 \%$, leukocyte count $8,700 / \mu$ (neutrophils $82 \%$, eosinophils $2 \%$, monocytes $8 \%$, lymphocytes $8 \%$ ), and platelet count $33.1 \times 10^{4} / \mu$ l. The total urinary protein for 24 hours was $1.2 \mathrm{~g}$, and the urine sediment showed 3-5 red blood cell casts per high power field. The serum total protein was $6.6 \mathrm{~g} / \mathrm{dl}$, albumin $3.2 \mathrm{~g} / \mathrm{dl}$, blood urea nitrogen $40.5 \mathrm{mg} / \mathrm{dl}$, creatinine 2.5 $\mathrm{mg} / \mathrm{dl}$, total bilirubin $0.3 \mathrm{mg} / \mathrm{dl}$, aspartate aminotransferase 20 IU/l, alanine aminotransferase $20 \mathrm{IU} / 1$, alkaline phosphatase $116 \mathrm{IU} / \mathrm{l}$, and lactic dehydrogenase $388 \mathrm{IU} / \mathrm{l}$. Serum IgG was $1,814 \mathrm{mg} / \mathrm{dl}$, IgA $221 \mathrm{mg} / \mathrm{dl}$, and IgM $120 \mathrm{mg} / \mathrm{dl}$. The rheumatoid factor was negative, but antinuclear antibody (ANA) was positive $(\times 160)$ with a speckled pattern. Serum anti-double stranded DNA antibody, anti-Sm antibody, anti-SS-A antibodies, and anti-SS-B antibodies were negative. Serum C3 was 70 $\mathrm{mg} / \mathrm{dl}$ (normal range, $60-116 \mathrm{mg} / \mathrm{dl}$ ), C4 $34.9 \mathrm{mg} / \mathrm{dl}(15-44$ $\mathrm{mg} / \mathrm{dl}$ ), and CH50 $39 \mathrm{CH} 50 \mathrm{U} / \mathrm{ml}$ (30-45 CH50U/ml). Circulating immune complexes were not detected by the C1q-binding assay. Serum cryoglobulins were negative. P-ANCA were detected by indirect immunofluorescence staining, and the titer of anti-myeloperoxidase (MPO) antibodies, which was measured by the ELISA method, was $23 \mathrm{EU} / \mathrm{ml}$ (normal; less than 10 $\mathrm{EU} / \mathrm{ml}$ ). Both cytoplasmic ANCA (C-ANCA) and antiglomerular basement membrane antibodies were negative. The titer of tumor necrosis factor- $\alpha$ (TNF- $\alpha$ ) measured by ELISA was less than $5 \mathrm{pg} / \mathrm{ml}$. Streptococcal pneumoniae was detected by a sputum culture. The chest X-ray disclosed diffuse infiltrations at the bilateral lower lungs.

Since the patient's renal function worsened rapidly within 10 days of admission and oliguria (urine volume 100 to $300 \mathrm{ml} /$ $24 \mathrm{~h}$ ) was noted, hemodialysis was started on July 10, 1991. There were no pre-renal factors causing acute renal failure such as dehydration or hypotension. Post-renal factors causing oliguria were not detected by urological examinations. For the lung disease, the patient was treated with an antibiotic (ofloxacin). Since his productive cough and the infiltrations of bilateral lower lungs disappeared 7 days after this treatment, he was finally diagnosed as having bacterial pneumonia.

A needle renal biopsy was performed on August 2, 1991. Light microscopic examination demonstrated that almost all the glomeruli accompanied cellular crescents, and the glomerular tufts were collapsed. Segmental necrotic lesions were found in some glomeruli. The interstitium was diffusely edematous, and tubular atrophy and infiltrations of small round cells were observed focally. The tubular epithelial cells often showed vacuolar degeneration (Fig. 1). Immunofluorescence microscopy revealed $2+$ staining for $\mathrm{IgG}, 1+$ staining for $\kappa, \lambda$, and $\mathrm{C} 3$ over the mesangial area and along peripheral capillary walls in a granular pattern (Fig. 2), and 2+ staining for fibrinogen in crescents. Ultrathin sections for electron microscopy were stained with periodic acid silver methenamine (PASM) as described previously (9). Scattered deposits were shown mainly in the paramesangial area, and partly in the glomerular capillary walls (Fig. 3).

Based on these findings, the patient was diagnosed as having rapidly progressive glomerulonephritis due to diffuse immune complex type crescentic glomerulonephritis with the develop-
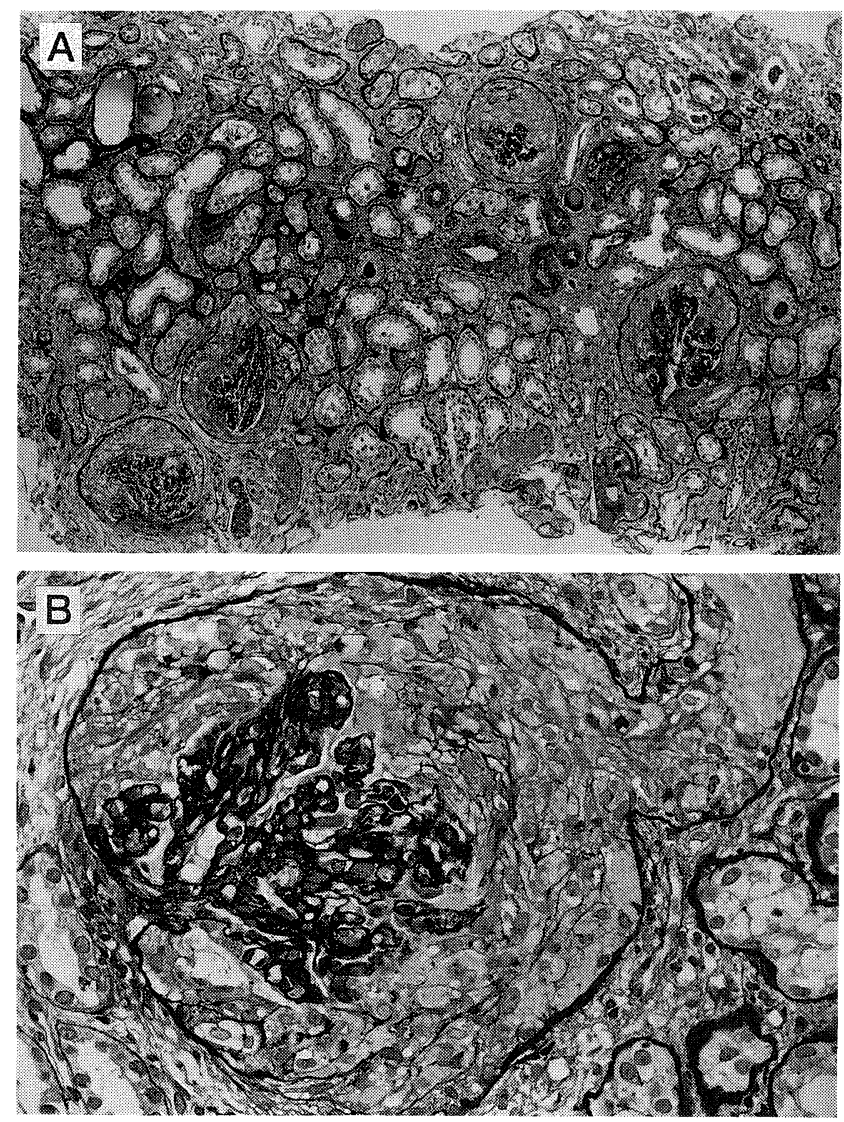

Fig. 1. A. Light microscopic examination demonstrates that almost all of the glomeruli accompanied the cellular crescent, and the interstitium is edematous with infiltration of small round cells (PAS stain, $\times 40$ ). B. The glomerular tufts are compressed by extensive crescent formation (PAS stain, $\times 400$ ).

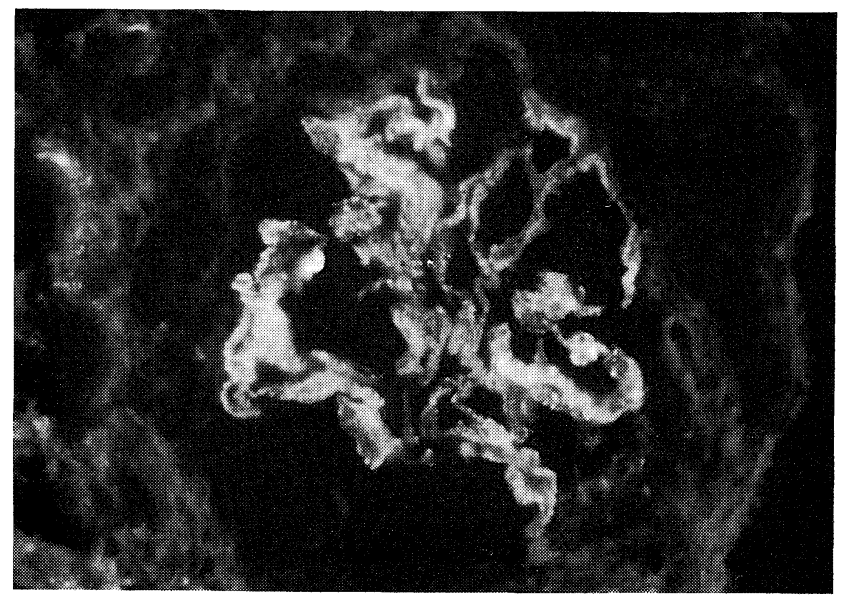

Fig. 2. Immunofluorescence microscopy reveals $2+$ staining for IgG over the mesangial area and along peripheral capillary walls in a granular pattern.

ment of serum P-ANCA. He was treated with plasma exchange $(5,000 \mathrm{ml} /$ day, for 3 days $)$ and prednisolone $(60 \mathrm{mg} /$ day for 6 weeks). In November 1991, P-ANCA was negative and the titer 


\section{Immune Complex Type Crescentic GN \& P-ANCA}

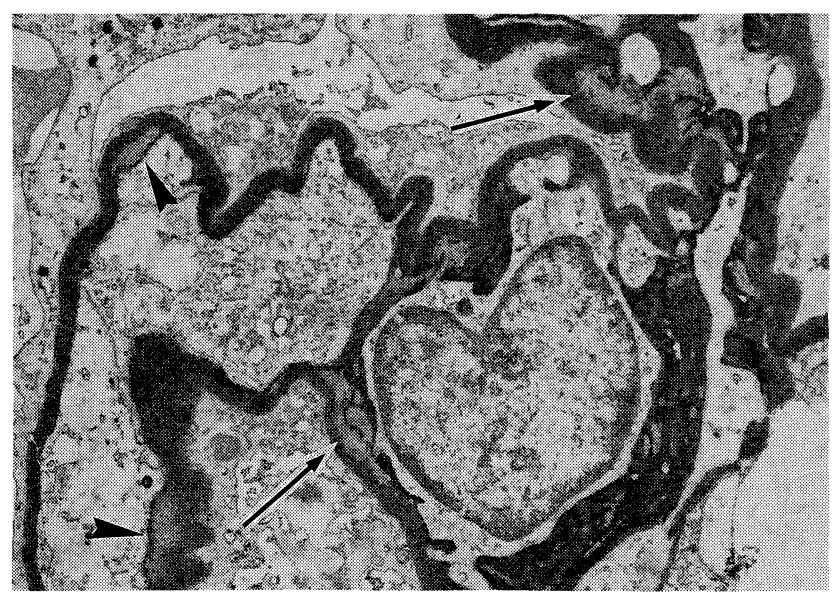

Fig. 3. Electron microscopy displays scattered deposits mainly in the paramesangial area (arrows) and partly in the glomerular capillary walls (arrow heads) (PASM stain, $\times 2,500$ ).

of anti-MPO antibodies was less than $10 \mathrm{EU} / \mathrm{ml}$. Unfortunately, the patient could not recover from renal failure. Prednisolone was tapered and then stopped in December 1991. Hemodialysis has been continued twice a week since July 1992.

\section{Discussion}

This patient developed rapidly progressive renal dysfunction due to immune complex type crescentic glomerulonephritis. Serological studies showed P-ANCA and anti-MPO antibodies in his serum.

It is known that many diseases cause diffuse crescentic glomerulonephritis, and that the pathogenetic mechanisms of crescent formation are heterogeneous $(1,2)$. Idiopathic crescentic glomerulonephritis is generally classified into three subsets by immunohistological criteria, 1) anti-glomerular basement membrane antibody-mediated type, 2) immune complex-mediated type, and 3) pauci-immune type. The second type of crescentic glomerulonephritis is identified by extensive glomerular deposits of IgG and/or IgM, often, but not always, accompanied by $\mathrm{C} 3$ depositions in a granular pattern $(1,2)$. The morphological features of the present patient were compatible with those of immune complex type crescentic glomerulonephritis including a form of lupus nephritis. Because this patient could not be diagnosed as SLE from the 1982 revised criteria for the classification of SLE (10), the relationship between ANA and crescent formation was unclear. Furthermore, the laboratory findings were not typical; serum $\mathrm{C} 3, \mathrm{C} 4$, and $\mathrm{CH} 50$ were within normal limits, and circulating immune complexes were not detected. These facts suggest that the crescent formation in the present case may not be mediated by immune complex deposits.

In 1982, ANCA were first reported by Davies et al (11) in 8 patients with pauci-immune necrotizing glomerulonephritis. Van der Woude et al (12) demonstrated that 25 out of 27 patients with active Wegener's granulomatosis had high titers of ANCA, and that ANCA is useful as a marker of disease activity. Since
1988, ANCA have been classified into cytoplasmic(C)- and perinuclear(P)-ANCA by indirect immunofluorescent microscopy using alcohol fixed neutrophils as substrata $(3,6)$. C-ANCA are generally specific for proteinase $3(13,14)$, and CANCA are frequently detected in patients with Wegener's granulomatosis $(15,16)$. On the other hand, P-ANCA usually react with myeloperoxidase (6), and P-ANCA appear in a broad spectrum of patients, ranging from those with pauci-immune crescentic glomerulonephritis, polyarteritis nodosa, and ChurgStrauss syndrome, to collagen diseases including SLE (3-5, 17). Therefore, Jennette et al $(3,5)$ and Falk (4) proposed that ANCA-positive glomerulonephritis and systemic necrotizing vasculitis are termed ANCA-associated diseases.

ANCA are consistently negative in immune complex type crescentic glomerulonephritis (1). However, Jennette et al (3) described that three out of 55 patients with non-lupus immune complex type crescentic glomerulonephritis had positive ANCA values by ELISA. One of the three patients had diffuse necrotizing and crescentic glomerulonephritis, $2+$ granular IgG deposition by immunohistology, and scattered glomerular dense deposits by electron microscopy. Although the detailed clinical and laboratory data of their patient were not available, the morphological features of this case were similar to those of the present case. Therefore, it is clear that some patients with immune complex type crescentic glomerulonephritis are considered to have ANCA, and ANCA may have an important role in the pathogenesis of crescent formation in immune complex type crescentic glomerulonephritis.

ANCA is thought to activate neutrophils and mononuclear phagocytes directly, and to play an important role in the development of necrotizing and crescentic glomerulonephritis, and necrotizing systemic vasculitis. In the clinical findings, it is commonly noted that patients with ANCA develop crescentic glomerulonephritis after upper respiratory or influenza-like illness $(1,2)$. Jennette et al (3) proposed that a trigger event, such as a viral infection, primes neutrophils in patients with ANCA; the primed neutrophils are activated by ANCA, and they cause vascular injury. Ewert et al (18) demonstrated that neutrophils stimulated by anti-MPO antibodies with TNF- $\alpha$ and endotoxin damage human endothelial cells in vitro. They stated that the cytokine dependency of this effect supports the clinical finding that patients with ANCA may have no symptom until there is unrelated activation of the immune system such as that which occurs with viral infections. Therefore, the event of acute pneumonia in the present patient may have played an important role in the production of some cytokines, and may have been a trigger of crescent formation, although TNF- $\alpha$ was not detected in this patient. Further case studies will be necessary to clarify the mechanism of crescent formation associated with ANCA in immune complex type crescentic glomerulonephritis.

Acknowledgement: We are grateful to Dr. Yoshikazu Sado of the Division of Immunology, Shigei Medical Research Hospital, Okayama, who measured anti-glomerular basement membrane antibody by ELISA, and Dr. Yoshihiro Arimura of the First Department of Internal Medicine, School of Medicine, 


\section{Komatsuda et al}

Kyorin University, Tokyo, who measured anti-myeloperoxidase antibody by ELISA.

\section{References}

1) Glassock RJ, Adler SG, Ward HJ, and Cohen AH. Primary glomerular disease. in: The Kidney, fourth edition, Brenner BM, Rector FC, Eds, WB Saunders Company, Philadelphia, 1991, p. 1182.

2) Salant DJ. Nephrology forum. Immunopathogenesis of crescentic glomerulonephritis and lung purpura. Kidney Int 32: 408, 1987.

3) Jennette JC, Wilkman AS, Falk RJ. Anti-neutrophil cytoplasmic autoantibody-associated glomerulonephritis and vasculitis. Am J Pathol 135: 921, 1989.

4) Falk RJ. Nephrology forum. ANCA-associated renal disease. Kidney Int 38: $998,1990$.

5) Jennette JC, Falk RJ. Anti-neutrophil cytoplasmic autoantibodies and associated diseases: A review. Am J Kidney Dis 15: 517, 1990.

6) Falk RJ, Jennette JC. Anti-neutrophil cytoplasmic autoantibodies with specificity for myeloperoxidase in patients with systemic vasculitis and crescentic glomerulonephritis. N Engl J Med 318: 1651, 1988.

7) Lockwood CM, Bowman C, Bakes D, Pressery A, Dash A. Autoimmunity and glomerulonephritis. Adv Nephrol 16: 291, 1987.

8) Jayne DRW, Marshall PD, Jones SJ, Lockwood CM. Autoantibodies to GBM and neutrophil cytoplasm in rapidly progressive glomerulonephritis. Kidney Int 37: 965, 1990.

9) Yasuda $\mathrm{T}$, Nakamoto $\mathrm{Y}$, Miki K, Imai H, Asakura K, Miura AB.
Collagenofibrotic glomerulopathy. in: Collagenofibrotic Glomerulonephropathy, Arakawa M, Yamanaka N, Eds. Nishimura Co., Ltd, Niigata, 1991, p. 31.

10) Tan EM, Cohen AS, Fries JF, et al. The 1982 revised criteria for the classification of systemic lupus erythematosus. Arthritis Rheum 25: 1271, 1982.

11) Davies DJ, Moran JE, Niall JF, Ryan GB. Segmental necrotizing glomerulonephritis with antineutrophil antibody: Possible arbovirus aetiology? Br Med J 285: 606, 1982.

12) Van der Woude FJ, Rasmussen $N$, Lobatto $S$, et al. Autoantibodies against neutrophils and monocytes: Tool for diagnosis and marker of disease activity in Wegener's granulomatosis. Lancet 1: 425, 1985.

13) Ludemann J, Utecht B, Gross WL. Anti-neutrophil cytoplasmic antibodies in Wegener's granulomatosis recognize an elastinolytic enzyme. J Exp Med 171: 357, 1990.

14) Jennette JC, Hoidal JR, Falk RJ. Specificity of anti-neutrophil cytoplasmic autoantibodies for proteinase 3. Blood 75: 2263, 1990.

15) Gans ROB, Goldschmeding R, Donker AJM, et al. Neutrophil cytoplasmic autoantibodies and Wegener's granulomatosis. Lancet 1: 269, 1989.

16) Specks U, Wheatley CL, McDonald TJ, Rohrback MS, DeRemee RA. Anti-cytoplasmic antibodies in the diagnosis and follow-up of Wegener's granulomatosis. Mayo Clin Proc 64: 28, 1989.

17) Gross WL, Schmitt WH, Csernok E. Antineutrophil cytoplasmic autoantibody-associated diseases: A rheumatologist's perspective. Am J Kidney Dis 18: 175, 1991.

18) Ewert BH, Jennette JC, Falk RJ. Anti-myeloperoxidase antibodies stimulate neutrophils to damage human endothelial cells. Kidney Int 41: 375, 1992. 